\title{
Online automatic tuning and control for fed-batch cultivation
}

\author{
Zita I. T. A. Soons - Gerrit van Straten • \\ Leo A. van der Pol · Anton J. B. van Boxtel
}

Received: 17 October 2007 / Accepted: 3 December 2007/Published online: 21 December 2007

(C) The Author(s) 2007

\begin{abstract}
Performance of controllers applied in biotechnological production is often below expectation. Online automatic tuning has the capability to improve control performance by adjusting control parameters. This work presents automatic tuning approaches for model reference specific growth rate control during fed-batch cultivation. The approaches are direct methods that use the error between observed specific growth rate and its set point; systematic perturbations of the cultivation are not necessary. Two automatic tuning methods proved to be efficient, in which the adaptation rate is based on a combination of the error, squared error and integral error. These methods are relatively simple and robust against disturbances, parameter uncertainties, and initialization errors. Application of the specific growth rate controller yields a stable system. The controller and automatic tuning methods are qualified by simulations and laboratory experiments with Bordetella pertussis.
\end{abstract}

Keywords Online automatic tuning .

Fed-batch cultivation - Specific growth rate .

Bordetella pertussis $\cdot$ Stability

\section{List of symbols}

$a, b, c, d \quad$ constants for dual substrate model for B. pertussis

Z. I. T. A. Soons - G. van Straten - A. J. B. van Boxtel Systems and Control Group, Wageningen University, P.O. box 17, 6700 AA Wageningen, The Netherlands

Z. I. T. A. Soons $(\bowtie) \cdot$ L. A. van der Pol Netherlands Vaccine Institute, P.O. box 457,

3720 AL Bilthoven, The Netherlands

e-mail: zita.soons@wur.nl $a_{1}, \quad a_{2}, \ldots, \quad$ constants for disturbances on model

$a_{7} \quad$ parameters

$A, B, C \quad$ system matrices for dual substrate model for B. pertussis

$C_{\mathrm{G}}, C_{\mathrm{G}}^{\mathrm{in}} \quad$ glutamate concentration in the medium and in the feed $\left(\mathrm{mmol} \mathrm{l}^{-1}\right)$

$C_{\mathrm{L}}, C_{\mathrm{L}}^{\text {in }} \quad$ lactate concentration in the medium, respectively in the feed $\left(\mathrm{mmol} \mathrm{l}^{-1}\right)$

$C_{\mathrm{N}} \quad$ nominal value of controller $\left(\mathrm{mmol} \mathrm{l}^{-1}\right)$

$C_{X} \quad$ biomass concentration (OD)

$c_{1}, c_{2}, c_{3}$ constants for the choice of the adaptation mechanisms

DO, dissolved oxygen in the medium and

$\mathrm{DO}_{\text {sensor }} \quad$ measured by the sensor $\left(\mathrm{mmol} \mathrm{l}^{-1}\right)$

$\mathrm{DO}_{\text {set }} \quad$ set point dissolved oxygen $\left(\mathrm{mmol} \mathrm{l}^{-1}\right)$

$e$ error between reference model and process, $\mu_{\text {ref }}-\hat{\mu}\left(\mathrm{h}^{-1}\right)$

$E \quad$ mean absolute error $\left(\mathrm{h}^{-1}\right)$

$F \quad$ state feedback matrix

$F_{\mathrm{G}+\mathrm{L}}^{\mathrm{in}} \quad$ total substrate feed rate (glutamate + lactate) $\left(1 \mathrm{~h}^{-1}\right)$

$k \quad$ time instant

$k_{L} a \quad$ oxygen transfer coefficient $\left(\mathrm{h}^{-1}\right)$

$K_{\mathrm{C}}, K_{I}, K_{P} \quad$ controller gains

$K_{\mathrm{G}}, K_{\mathrm{L}} \quad$ monod constant on glutamate and lactate (mmol $\left.1^{-1}\right)$

$m_{\mathrm{G}}, \quad m_{\mathrm{L}}, \quad$ maintenance coefficient on glutamate, lactate

$m_{\mathrm{O}} \quad$ and oxygen $\left(\mathrm{mmol} \mathrm{OD}{ }^{-1} \mathrm{~h}^{-1}\right.$ )

$N \quad$ length of moving window

$O \quad$ oscillations measure $\left(\mathrm{h}^{-1}\right)$

$\mathrm{O}_{2}^{\mathrm{h}}, \mathrm{O}_{2}^{\mathrm{in}} \quad$ oxygen concentration in headspace, and in incoming air (liquid phase) $\left(\mathrm{mmol} \mathrm{1}^{-1}\right)$

OD optical density at $590 \mathrm{~nm}$

OUR, oxygen uptake rate, oxygen transfer rate OTR $\quad\left(\mathrm{mmol} \mathrm{l} \mathrm{l}^{-1} \mathrm{~h}^{-1}\right)$ 


\begin{tabular}{|c|c|}
\hline$p, p^{\mathrm{d}}$ & $\begin{array}{l}\text { model parameters, model parameters with } \\
\text { disturbances }\end{array}$ \\
\hline$t$ & cultivation time $(\mathrm{h})$ \\
\hline$V$ & liquid volume (1) \\
\hline$u, x, y$ & inputs, states, output \\
\hline$Y_{\mathrm{G} 1}, Y_{\mathrm{G} 2}$ & $\begin{array}{l}\text { biomass yield on glutamate over pathway } 1 \text {, } \\
\text { respectively, pathway } 2\left(\mathrm{OD} \mathrm{mmol}{ }^{-1}\right)\end{array}$ \\
\hline$Y_{\mathrm{L}}, Y_{\mathrm{O}}$ & $\begin{array}{l}\text { biomass yield on lactate, respectively, on } \\
\text { oxygen }(\mathrm{OD} \mathrm{mmol}\end{array}$ \\
\hline$\beta$ & adaptation rate \\
\hline$\gamma_{1}, \gamma_{2}$ & tuning parameters for $\mu$ control \\
\hline$\varepsilon$ & $\begin{array}{l}\text { error between set point and process, } \\
\mu_{\text {set }}-\hat{\mu}\left(\mathrm{h}^{-1}\right)\end{array}$ \\
\hline$\mu, \mu_{\text {set }}$ & $\begin{array}{l}\text { specific growth rate, set point specific growth } \\
\text { rate }\left(h^{-1}\right)\end{array}$ \\
\hline$\mu_{\mathrm{enh}}, \mu_{\max }$ & $\begin{array}{l}\text { enhanced, maximum specific growth rate } \\
\left(\mathrm{h}^{-1}\right)\end{array}$ \\
\hline$\tau_{I}$ & reset time for dissolved oxygen control (h) \\
\hline$\tau_{\text {sensor }}$ & response time for dissolved oxygen sensor $(h)$ \\
\hline$\wedge$,EKF & estimated values \\
\hline
\end{tabular}

\section{Introduction}

Unacceptably sluggish or oscillatory controllers are generally classified as either "fair" or "poor" while controllers with minor performance deviations are classified as "acceptable" or "excellent". Desbourough and Miller [1] state that $32 \%$ of 26,000 controllers investigated in industry is classified as "poor" or "fair". Only one third of the controllers were classified as acceptable performers and two thirds had significant improvement opportunity. Figure 1 shows an example of an experimental run for specific growth rate control in vaccine production on lab-scale. The strong oscillations show the poor performance and indicate the relevance of automatic tuning in this field of application.

In biopharmaceutical production micro-organisms are cultivated in batch or fed-batch systems. Most vaccines, such as whooping cough, are produced in batch cultivation. Production of pertussis toxin (which is one of the important antigens in the vaccine against infection with whooping cough) is strongly growth-associated [2, 3]. Deviations in specific growth rate will therefore lead to deviations in antigen levels and vaccine quality. To obtain a high quality-vaccine and to ensure batch-to-batch consistency, it is important to control the specific growth rate at a constant level. Soons et al. [4] developed a specific growth rate controller by combining a reference model and a cultivation model. The result is a control law, which is adaptive for changes in volume and biomass. It was shown that the choice of the reference model is a main factor determining the performance of the specific growth rate controller.

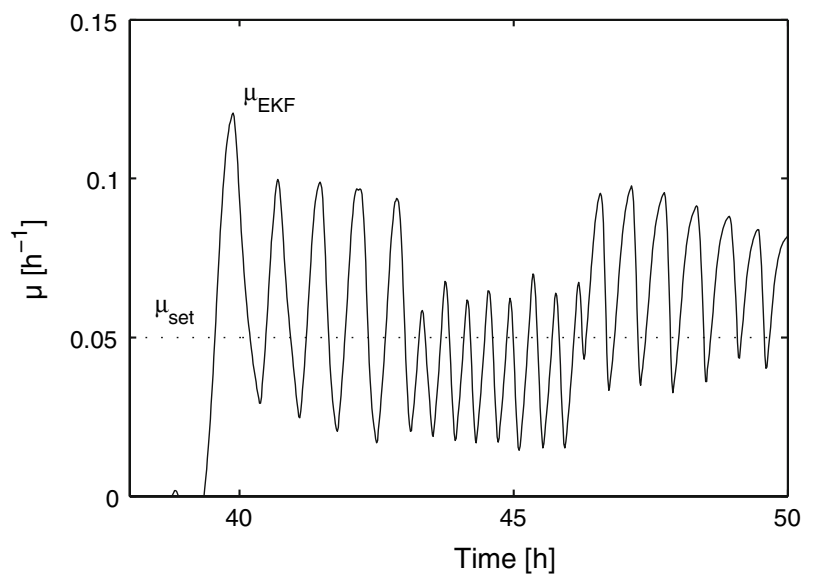

Fig. 1 Illustration of a process showing poor performance. Fed-batch cultivation with specific growth rate control for an experimental run of vaccine production on lab-scale [5]

Apart from the reference model, biological behavior is essential for the controller performance and as we know biological behavior is not perfectly known. Despite good performance in [4], the method does not correct for residual errors due to model-process mismatches [5]. Changes in the system, inaccurate knowledge about kinetics, varying properties of micro-organisms and response times of sensor systems are reasons for modelprocess mismatches. Online automatic tuning has the capability to deal with the mismatches and to improve controller performance by adjusting the controller parameters [6].

The aim of this paper is to investigate schemes for automating and to speed up the tuning procedures. To this end, the specific growth rate controller for fed-batch cultivation developed in [4] is extended with an automatic tuning method that is driven by the persistence of the residual errors. A direct method (in which the adjustment rule makes directly updates of the controller parameters) is preferred over an indirect method (in which process parameters are estimated to update controller parameters) as in this way a degree of imperfection of the model for model-based control can be handled. Moreover, probing should be avoided, because it may affect the critical variables in biopharmaceutical production.

Although a priori closed loop stability is hard to guarantee for a fed-batch system in which the biomass increases exponentially, stability of the control loops under application of the designed control law is evaluated.

This paper is organized as follows. First a brief description of the process and control system is given. Next, controller evaluation criteria are defined. Then, a short literature review and a textbook method for online automatic tuning using the MIT rule are presented. Due to unsatisfactory results of this method three algorithms are derived 
and evaluated by simulations in Sect. "Online automatic tuning methods". Also, stability of the closed loop is considered. Finally, the two best methods are tested in laboratory experiments for B. pertussis in Sect. "Experimental Results".

\section{Process description}

Process model

A process model for vaccine production of $B$. pertussis is used to evaluate the controllers with automatic tuning. The production is performed in fed-batch cultivation, in which the specific growth rate is controlled to achieve a higher level of batch-to-batch consistency. Growth of B. pertussis is limited by two substrates [7] and is modeled by the following dual substrate model assuming Monod kinetics and oxygen excess $[4,8-10]$ during fed-batch cultivation (Eqs. 1-13). The changes of dissolved oxygen in time are equal to the oxygen transfer rate minus the oxygen uptake rate minus the dilution due to the substrate feed rate.

$$
\begin{aligned}
& \mu\left(C_{\mathrm{G}}, C_{\mathrm{L}}\right)=\mu_{\text {max }} f_{\mathrm{G}}\left(C_{\mathrm{G}}\right)+\mu_{\mathrm{enh}} f_{\mathrm{G}}\left(C_{\mathrm{G}}\right) f_{\mathrm{L}}\left(C_{\mathrm{L}}\right) \\
& f_{\mathrm{G}}\left(C_{\mathrm{G}}\right)=\frac{C_{\mathrm{G}}}{K_{\mathrm{G}}+C_{\mathrm{G}}} \quad \text { and } \quad f_{\mathrm{L}}\left(C_{\mathrm{L}}\right)=\frac{C_{\mathrm{L}}}{K_{\mathrm{L}}+C_{\mathrm{L}}}
\end{aligned}
$$

$$
\frac{\mathrm{d} V}{\mathrm{~d} t}=F_{G+L}^{\mathrm{in}}
$$

$$
\begin{aligned}
\frac{\mathrm{d} C_{X}}{\mathrm{~d} t} & =\left(\mu-\frac{F_{G+L}^{\mathrm{in}}}{V}\right) C_{X} \\
& =\left(\mu_{\max } f_{\mathrm{G}}\left(C_{\mathrm{G}}\right)+\mu_{\mathrm{enh}} f_{\mathrm{G}}\left(C_{\mathrm{G}}\right) f_{\mathrm{L}}\left(C_{\mathrm{L}}\right)-\frac{F_{G+L}^{\mathrm{in}}}{V}\right) C_{X}
\end{aligned}
$$

$$
\begin{aligned}
\frac{\mathrm{d} C_{\mathrm{G}}}{\mathrm{d} t}= & \frac{F_{G+L}^{\mathrm{in}}}{V}\left(C_{\mathrm{G}}^{\mathrm{in}}-C_{\mathrm{G}}\right) \\
& -\left(\frac{\mu_{\mathrm{max}} f_{\mathrm{G}}\left(C_{\mathrm{G}}\right)}{Y_{\mathrm{G} 1}}-\frac{\mu_{\mathrm{enh}} f_{\mathrm{G}}\left(C_{\mathrm{G}}\right) f_{\mathrm{L}}\left(C_{\mathrm{L}}\right)}{Y_{\mathrm{G} 2}}+m_{\mathrm{G}}\right) C_{X}
\end{aligned}
$$

$$
\frac{\mathrm{d} C_{\mathrm{L}}}{\mathrm{d} t}=\frac{F_{G+L}^{\mathrm{in}}}{V}\left(C_{\mathrm{L}}^{\mathrm{in}}-C_{\mathrm{L}}\right)-\left(\frac{\mu_{\mathrm{enh}} f_{\mathrm{G}}\left(C_{\mathrm{G}}\right) f_{\mathrm{L}}\left(C_{\mathrm{L}}\right)}{Y_{L 2}}+m_{\mathrm{L}}\right) C_{X}
$$

$\frac{\mathrm{dDO}}{\mathrm{d} t}=\mathrm{OTR}-\mathrm{OUR}-\frac{F_{G+L}^{\mathrm{in}}}{V} \cdot \mathrm{DO}$

The oxygen uptake rate (OUR) is the sum of oxygen used for growth $\left(\mu / Y_{\mathrm{O}}\right)$ and oxygen used for maintenance $\left(m_{\mathrm{O}}\right)$ :

OUR $=\left(\frac{\mu}{Y_{o}}+m_{o}\right) C_{x}$

The bioreactor is aerated using headspace aeration only. The liquid phase oxygen concentration $\left(\mathrm{O}_{2}^{\mathrm{h}}\right)$ in equilibrium with the gas phase in the headspace following Henry's law [9], the dissolved oxygen (DO), and the oxygen transfer coefficient $k_{L} a$ determine the oxygen transfer rate (OTR):

$\mathrm{OTR}=k_{l} a \cdot\left(O_{2}^{h}-\mathrm{DO}\right)$

The dynamics of oxygen is much faster than the dynamics of the other relevant processes (e.g. biomass, substrates) [11] and the contribution of the dilution term is small (third term Eq. 7) compared to the rate of change of dissolved oxygen. As a consequence, Eq. 7 is considered in steadystate and OUR is calculated every minute during the experiment using Eq. 10:

OUR $\approx$ OTR

$\mathrm{O}_{2}^{h}$ is assumed equal to $\mathrm{O}_{2}^{\text {in }}$ because of the high aeration rate:

$O_{2}^{h} \approx O_{2}^{\text {in }}$

The DO sensor has a response time around $20 \mathrm{~s}$, and is modeled as a first order system:

$\frac{\mathrm{dDO}_{\text {sensor }}}{\mathrm{d} t}=-\frac{1}{\tau_{\text {sensor }}} \cdot \mathrm{DO}_{\text {sensor }}+\frac{1}{\tau_{\text {sensor }}} \cdot \mathrm{DO}$

Dissolved oxygen is controlled by changing the incoming oxygen fraction $\left(\mathrm{O}_{2}^{\text {in }}\right)$ in an oxygen/air/nitrogen mixture. The controller is based on an already installed PI controller:

$$
\begin{aligned}
O_{2}^{\text {in }}= & K_{C} \cdot\left(\mathrm{DO}_{\text {set }}-\mathrm{DO}_{\text {sensor }}\right) \\
& +\int_{0}^{t} \frac{K_{C}}{\tau_{I}} \cdot\left(\mathrm{DO}_{\text {set }}-\mathrm{DO}_{\text {sensor }}\right) \mathrm{d} t+C_{\mathrm{N}}
\end{aligned}
$$

Specific growth rate control system

Figure 2 gives an overview of the proposed control system for fed-batch cultivation. It is the same as the scheme presented by Soons et al. [4], except that it is extended with an automatic tuning method. The control purpose is to regulate the specific growth rate $(\mu)$ to a desired value by

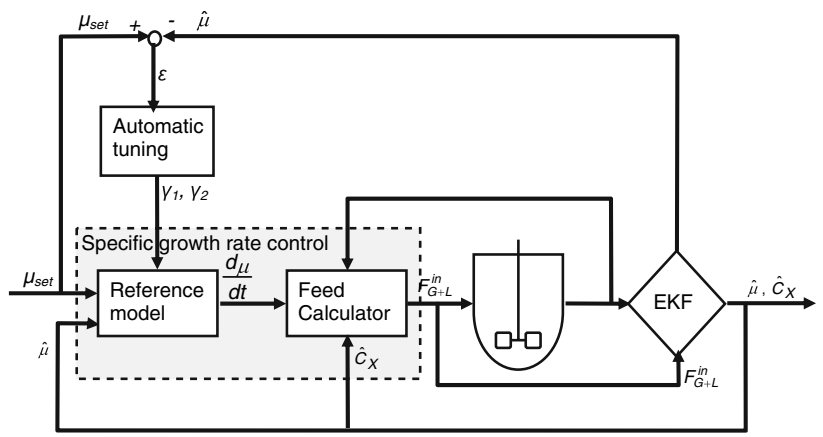

Fig. 2 The control system for adaptive specific growth rate control with automatic tuning 
adding a feed with limiting substrates. The grey block defines the specific growth rate controller, which will be briefly reviewed below.

The definition of the specific growth rate controller given in [4] started from a second order reference model:

$\frac{\mathrm{d} \hat{\mu}}{\mathrm{d} t}=\frac{\mu_{\mathrm{set}}-\hat{\mu}}{\gamma_{1}}+\int_{0}^{t} \frac{\mu_{\mathrm{set}}-\hat{\mu}}{\gamma_{2}} \mathrm{~d} t$

where the tuning parameters $\gamma_{1}$ and $\gamma_{2}$ determine the convergence speed of the controller and therefore controller performance. The reference model is stable if $\gamma_{1}$ and $\gamma_{2}$ are strictly positive numbers. Combination with the process model yields an adaptive "PI" controller:

$$
\begin{aligned}
F_{G+L}^{\text {in }}= & \frac{a c+b d}{a C_{\mathrm{G}}^{\text {in }}+b C_{\mathrm{L}}^{\text {in }}} \hat{C}_{X} V+K_{P} \cdot\left(\mu_{\mathrm{set}}-\hat{\mu}\right) \\
& +\int_{0}^{K_{I}} K_{I} \cdot\left(\mu_{\mathrm{set}}-\hat{\mu}\right) \mathrm{d} t
\end{aligned}
$$

where the controller gains $K_{P}$ and $K_{I}$ are adjusted online to the changing volume:

$K_{P}=\frac{V}{\gamma_{1}\left(a C_{\mathrm{G}}^{\text {in }}+b C_{\mathrm{L}}^{\text {in }}\right)} \quad$ and $\quad K_{I}=\frac{V}{\gamma_{2}\left(a C_{\mathrm{G}}^{\text {in }}+b C_{\mathrm{L}}^{\text {in }}\right)}$

$a, b, c$, and $d$ are constants depending on the microorganism (in this work Bordetella pertussis [4]).

Biomass and specific growth rate measurements are often not available online. Therefore, an Extended Kalman Filter (EKF) estimates specific growth rate $(\hat{\mu})$ and biomass $\left(\hat{C}_{X}\right)$ using the measured oxygen uptake rate every minute $[4,12]$. This software sensor showed fast convergence and fitted well to the data (see Appendix 1 for an application on continuous cultivation). Therefore, for controller design the estimations of the EKF are taken as replacement of actual measurements $\left(\mu=\hat{\mu}\right.$ and $\left.C_{X}=\hat{C}_{X}\right)$.

Central point of this work is the extension of the specific growth rate controller (Eq. 15) with three methods for automatic tuning, which adapt the controller parameters $\gamma_{1}$ and $\gamma_{2}$ on the basis of the error in specific growth rate (see Fig. 2). So the approach does not require probing which can disturb the critical variables in biopharmaceutical production.

Table 1 Model parameters and initial conditions for simulations with the dual substrate model of B. pertussis

\section{Controller evaluation}

Evaluation methods

The model (Eqs. 1-13) is used to evaluate controller performance in simulations in Sect. "Online automatic tuning methods". To obtain realistic tests of the robustness of the controller and performance of the automatic tuning several disturbances have been introduced in the simulation as in [4]: time-varying or drifting kinetics, initialization errors on the controller tuning parameters, and noise. We assumed a drift plus a sinus with different frequencies on the model parameters, whereas in the controller, the model parameters $p$ were set constant:

$p^{d}=p+a_{1} t+a_{2} \sin \left(a_{3} \pi t+a_{4} \pi\right)+a_{5} \sin \left(a_{6} \pi t+a_{7} \pi\right)$

where $p^{d}$ are the disturbed parameters for $\mu_{\max }, \mu_{\mathrm{enh}}, Y_{G 1}$, $Y_{G 2}, Y_{L}, C_{G}^{\mathrm{in}}, C_{L}^{\mathrm{in}}, K_{G}$, and $K_{L}$. The parameters and initial values are given in Table 1, the disturbances on the parameters in Table 2.

In practice, dissolved oxygen measurements contain observation noise. In the simulations noise is introduced by adding white noise with an intensity proportional to OUR $(0.1$ initially to $3 \%$ at end). This intensity was chosen to mimic the observed fact that DO is more noisy toward the end:

$\mathrm{DO}_{\text {sensor }}^{m}\left(t_{k}\right)=\mathrm{DO}_{\text {sensor }}\left(t_{k}\right)+v\left(t_{k}\right)$

In addition to [4], controller tuning parameters $\gamma_{1}$ and $\gamma_{2}$ are initialized far below the required values, which results in underdamped behavior. When the specific growth rate

Table 2 Disturbances on the model parameters for B. pertussis

\begin{tabular}{lll}
\hline Disturbance parameter & \multicolumn{2}{l}{ Value } \\
\cline { 2 - 3 } & From & To \\
\hline$a_{1}$ & 0 & $\pm 1 \times 10^{-3} p$ \\
$a_{2}$ & 0 & $\pm 0.2 p$ \\
$a_{3}$ & 0 & 0.1 \\
$a_{4}$ & 0 & 1.2 \\
$a_{5}$ & 0 & $\pm 0.3 p$ \\
$a_{6}$ & 0 & 0 \\
$a_{7}$ & 0 & 1.2 \\
\hline
\end{tabular}

\begin{tabular}{lll}
\hline$V_{0}=3 l$ & $Y_{\mathrm{L}}=0.018 \mathrm{OD} \mathrm{mmol}^{-1}$ & $Y_{\mathrm{G} 1}=0.055 \mathrm{OD} \mathrm{mmol}^{-1}$ \\
$C_{X 0}=0.05 \mathrm{OD}$ & $m_{\mathrm{L}}=0 \mathrm{mmol} \mathrm{OD}^{-1} \mathrm{~h}^{-1}$ & $Y_{\mathrm{G} 2}=0.061 \mathrm{OD} \mathrm{mmol}^{-1}$ \\
$C_{\mathrm{G} 0}=10 \mathrm{mmol} \mathrm{l}^{-1}$ & $\mu_{\mathrm{max}}=0.12 \mathrm{~h}^{-1}$ & $m_{\mathrm{G}}=0 \mathrm{mmol} \mathrm{OD}^{-1} \mathrm{~h}^{-1}$ \\
$C_{\mathrm{G}}^{\mathrm{in}}=500 \mathrm{mmol} \mathrm{l}^{-1}$ & $\mu_{\mathrm{enh}}=0.055 \mathrm{~h}^{-1}$ & $Y_{\mathrm{O}}=0.041 \mathrm{OD} \mathrm{mmol}^{-1}$ \\
$C_{\mathrm{L} 0}=16.7 \mathrm{mmol} \mathrm{l}^{-1}$ & $K_{\mathrm{G}}=0.5 \mathrm{mmol} \mathrm{l}^{-1}$ & $m_{\mathrm{O}}=0.41 \mathrm{mmol} \mathrm{OD}^{-1} \mathrm{~h}^{-1}$ \\
$C_{\mathrm{L}}^{\text {in }}=835 \mathrm{mmol} \mathrm{l}^{-1}$ & $K_{\mathrm{L}}=0.5 \mathrm{mmol} \mathrm{l}^{-1}$ & $\hat{\mu}_{o}=0.16 \mathrm{~h}^{-1}$ \\
\hline
\end{tabular}




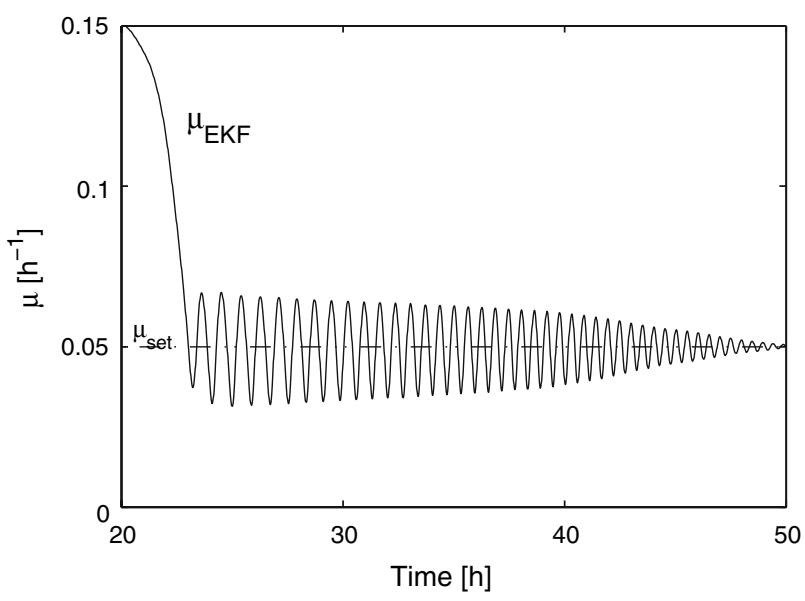

Fig. 3 Illustration of a simulation with specific growth rate control for poorly tuned and fixed $\gamma_{1}$ and $\gamma_{2}$

controller without automatic tuning method is applied with these settings, oscillations were present for almost $30 \mathrm{~h}$ (Fig. 3), underlining the need for additional adaptation. Experimental data showed similar profiles (Fig. 1). Additional simulations are performed with different values for the tuning parameters.

\section{Evaluation criteria}

To evaluate the significance of the automatic tuning system, two criteria are used. The criteria reflect the performance of the specific growth rate controller. In addition the simulation and experimental results are evaluated by comparing graphs.

Babuška et al. [13] used the following measures to detect a potential loss of performance. The first measure is the mean absolute error over the past $N$ points of a moving window at time point $k$ :

$E(k)=\frac{1}{N} \cdot \sum_{k-N}^{k}|\varepsilon(k)|$

The second is the oscillation measure and is defined by

$O(k)=\frac{1}{N} \cdot\left(\sum_{k-N}^{k}|\varepsilon(k)|-\sum_{k-N}^{k} \varepsilon(k)\right)$

where the error $\varepsilon$ is the set point minus the estimated specific growth rate $\left(\mu_{\text {set }}-\hat{\mu}\right)$.

\section{Literature}

\section{Literature review}

Åström and Wittenmark [14] give examples of modelbased adaptive control to adapt control parameters to changing process behavior. The adaptation of the controllers for a bioreactor as designed in [15-19] is also derived from a process model. This solution is limited by the accuracy of the models. Many adaptive controllers require measurement of the state variables such as biomass and substrates [20-24], which are often not available. Other approaches require identification of the system by introducing systematic disturbances $[13,25]$. Such "probing" should be avoided in cultivation systems for biopharmaceutical production as it may affect critical variables. Dagci et al. [6] used sliding mode control to adapt controller parameters. The authors show good control performance during continuous cultivation, but in fed-batch cultivation for vaccine production control performance was not satisfactory.

Classical automatic tuning approaches using PID control also require undesirable disturbances of the process [26]. Adaptation by a model predictive control approach proposed in [27] needs an open-loop model with the disadvantage that accuracy is not guaranteed. Another drawback of some of the reported solutions is the complex implementation in practice.

Industrial applications of adaptive control are often rule-based instead of model-based [28]. However, for control of complex and difficult industrial processes model-based control approaches have been proven the most effective among the different types of adaptive controllers [13].

Automatic tuning based on the mit rule

The task of the automatic tuning method is to pursue the best trade-off between tracking behavior, disturbance rejection, and stability. The automatic tuning method, therefore, should have the following abilities. At one hand, if control parameters $\gamma_{1}$ and $\gamma_{2}$ are initialized too small (Eq. 14), the automatic tuning method must increase the value of $\gamma_{1}$ and $\gamma_{2}$ to avoid underdamped behavior. On the other hand, the automatic tuning method should reduce the values of $\gamma_{1}$ and $\gamma_{2}$ if the error converges too slowly to zero. The automatic tuning method has to be derived so that a stable system is obtained in the sense that the error remains bounded.

In model-reference adaptive control according to the textbook of Aström and Wittenmark [14], the MIT rule is used to obtain adaptation. The error between the reference model and process output $\left(e=\mu_{\text {ref }}-\hat{\mu}\right)$ is the driver for the adaptation. Following the MIT rule the parameters $\gamma$ are adjusted such that a squared error is minimized:

$\frac{\mathrm{d} \gamma}{\mathrm{d} t}=-\beta \cdot e \cdot \frac{\partial e}{\partial \gamma}$ 
The vector $\frac{\partial e}{\partial \gamma}$ is the sensitivity of the error with respect to the controller parameter vector $\gamma$ (error sensitivity). The coefficient $\beta$ determines the adaptation rate and must be chosen so that the controller parameters change slower than the specific growth rate and dissolved oxygen, but fast enough to obtain adaptation.

\section{Online automatic tuning methods}

Adaptation based on the MIT rule is driven by the error $e$ which arises from set-point changes and disturbances. Systematic set-point changes must be avoided in cultivation systems for biopharmaceutical production as it may affect critical variables. As a consequence, in this work, adaptation is only driven by errors caused by disturbances. The model error $e$ equals the set-point error $\varepsilon$ and the reference model has become redundant.

The MIT rule requires knowledge of the error sensitivity, which is difficult to derive. Therefore, in literature approximations for the error sensitivity have been made [14]. Simulations with the outcome of several approximations gave poor results in the cultivation for vaccine production. Therefore alternative adaptation schemes are applied in the next section. Several (weighted) combinations of $\varepsilon, \varepsilon^{2}$, and $\int \varepsilon$ are investigated to approximate the sensitivity derivative:

$\frac{\partial \varepsilon}{\partial \gamma}=c_{1} \cdot 1+c_{2} \cdot \varepsilon+c_{3} \cdot \int_{0}^{t} \varepsilon$

Method 1: Adaptation based on the error

The most simple mechanism is assuming the sensitivity derivative to be one $[29,30]\left(c_{1}=-1, c_{2}=c_{3}=0\right)$.

$\frac{\mathrm{d} \gamma}{\mathrm{d} t}=\beta \cdot \varepsilon$

which appears to work well for adaptation of estimated process parameters. A test of Eq. 22 revealed that it is not a good mechanism for adaptation of controller parameters, because negative and positive errors will result in opposite adjustments of $\gamma$ and do not cancel oscillations.

Method 2: Adaptation based on the (intergral) error

In the second automatic tuning method the error sensitivity $\frac{\partial \varepsilon}{\partial \gamma}$ in Eq. 20 is replaced by a combination of the error and the integral error $\left(c_{1}=0, c_{2}=-1, c_{3}=-1\right)$.

$$
\begin{aligned}
& \frac{\mathrm{d} \gamma_{1}}{\mathrm{~d} t}=\varepsilon \cdot\left(\beta_{2} \varepsilon+\beta_{3} \int_{0}^{t} \varepsilon \mathrm{d} t\right) \\
& \frac{\mathrm{d} \gamma_{2}}{\mathrm{~d} t}=\varepsilon \cdot\left(\beta_{2} \varepsilon+\beta_{3} \int_{0}^{t} \varepsilon \mathrm{d} t\right)
\end{aligned}
$$

where $\beta_{2}$ and $\beta_{3}$ are a combination of $\beta$ and $c_{2}$ and $c_{3}$ $\left(\beta_{2}=\beta c_{2}\right.$ and $\left.\beta_{3}=\beta c_{3}\right)$. The automatic tuning method (Eq. 23) adapts control parameters $\gamma_{1}$ and $\gamma_{2}$ proportional to $\varepsilon^{2}$ and $\varepsilon \int \varepsilon$. So, $\gamma_{1}$ and $\gamma_{2}$ are predominately adapted when the quadratic error is large and/or when the error persists. The choice of the adaptation rate $\beta_{2}$ and $\beta_{3}$ is based on simulations. Too large values make adaptation faster than the change of the other variables in the system and make the adaptation mechanism react on instant errors instead of persistent errors, thereby avoiding the "basic" controller (Eq. 15) to counteract on these errors. Too small values of $\beta$ give slow adaptation, which results in the requirement for multiple tuning runs to upgrade performance. The choice of the adaptation rates for $\gamma_{1}$ and $\gamma_{2}$ is a choice for the designer of the controller, but simulations showed that distinction between the adaptation rates of $\gamma_{1}$ and $\gamma_{2}$ was not necessary.

This online automatic tuning method is applied to simulations of fed-batch cultivations in which $\gamma_{1}$ and $\gamma_{2}$ were initialized too small. By initiating the automatic tuning method at the start of the fed-batch, control performance was significantly upgraded within $5 \mathrm{~h}$ of cultivation (Fig. 4 shows a typical simulation). The mean absolute error and oscillation measure decreased fast after the start of the fedbatch resulting in upgraded controller performance (Fig. 4c, d). Compared to a simulation without adaptation of the controller tuning parameters, oscillations were attenuated five times faster (compare Figs. 3, 4).

Method 3: Adaptation based on the (squared) error

The third automatic tuning scheme consists of a combination of the error and the squared error, normalized by $\mu_{\mathrm{set}} \cdot\left(c_{1}=-\frac{1}{\mu_{\mathrm{set}}}, c_{2}=-\frac{1}{\mu_{\mathrm{set}}^{2}}, c_{3}=0\right)$.

$\frac{\mathrm{d} \gamma_{1}}{\mathrm{~d} t}=\beta_{1} \cdot \frac{\varepsilon}{\mu_{\text {set }}}+\beta_{2} \cdot \frac{\varepsilon^{2}}{\mu_{\text {set }}^{2}}$
$\frac{\mathrm{d} \gamma_{2}}{\mathrm{~d} t}=\beta_{1} \cdot \frac{\varepsilon}{\mu_{\text {set }}}+\beta_{2} \cdot \frac{\varepsilon^{2}}{\mu_{\text {set }}^{2}}$

where $\beta_{1}$ and $\beta_{2}$ are a combination of $\beta$ and $c_{1}$ and $c_{2}$ $\left(\beta_{1}=\beta c_{1}\right.$ and $\left.\beta_{2}=\beta c_{2}\right)$. Analogous to method 2 no distinction is made in $\beta_{i}$-values for the adaptation rates for $\gamma_{1}$ and $\gamma_{2}$. The automatic tuning method (Eq. 24) adapts control parameters $\gamma_{1}$ and $\gamma_{2}$ proportional to the error $\varepsilon$ and 

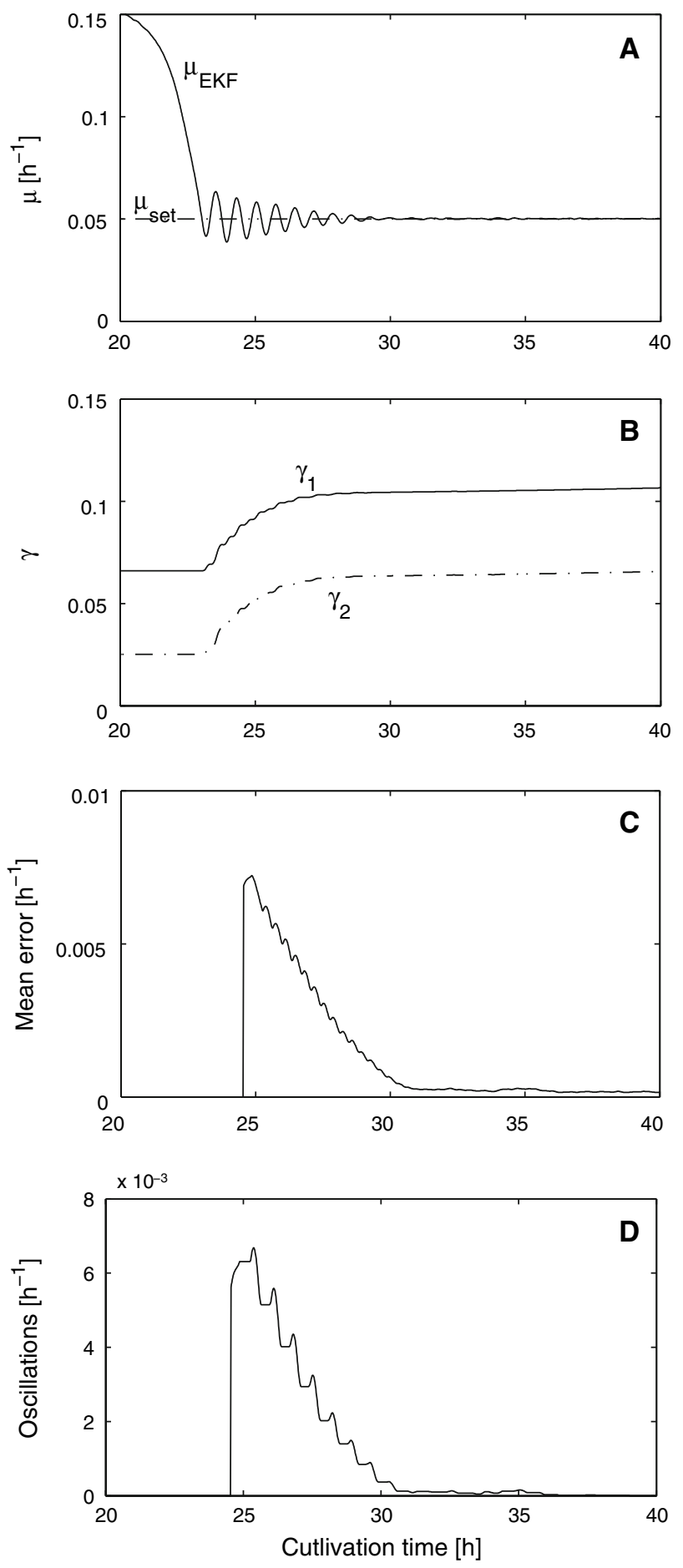

Fig. 4 Simulation results of specific growth rate control with automatic tuning based on the error and the integral error (method 2, Eq. 23). $\beta_{2}=225, \beta_{3}=550$. Fed-batch start at $t=22 \mathrm{~h}$. a Specific growth rate $(\mu)$. b Controller tuning parameters. c Mean absolute error. d Oscillation measure

proportional to the quadratic error $\varepsilon^{2}$, which is large if the error is large.

The fed-batch phase of the simulation in Fig. 5 started at $22 \mathrm{~h}$. At the same time the specific growth rate controller
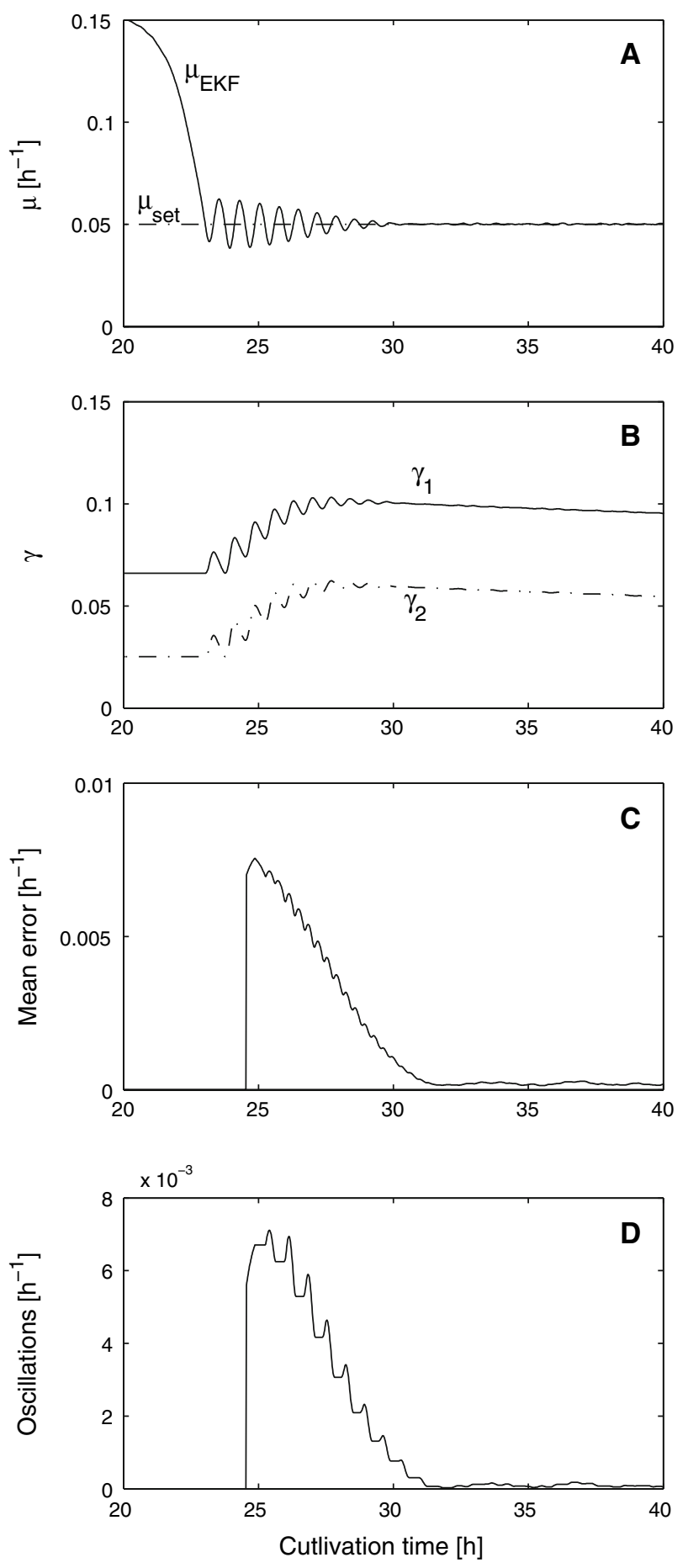

Fig. 5 Simulation results of specific growth rate control with automatic tuning based on the error and the squared error (method 3 , Eq. 24), $\beta_{1}=0.25, \beta_{2}=0.5$. Fed-batch start at $t=22 \mathrm{~h}$. a Specific growth rate $(\mu)$. b Controller tuning parameters. $\mathbf{c}$ Mean absolute error d Oscillation measure

was switched on with initial values of $\gamma_{1}$ and $\gamma_{2}$, which were chosen too low as in Fig. 3. The graph shows that these values resulted in underdamped responses (Fig. 5a), but the adjustment of $\gamma_{1}$ and $\gamma_{2}$ (Fig. 5b) was so strong that 
within $5 \mathrm{~h}$ the deviations were minimal and oscillations were cancelled (Fig. 5d). Figure 5c shows that controller performance was significantly upgraded with respect to the mean absolute error. Note that the mean absolute error and the oscillation measure were delayed $1.5 \mathrm{~h}$ to calculate their measures. Disturbances due to time-varying parameters and noise do not influence controller performance.

In other applications, the desired behavior can be acquired quickly by using this automatic tuning method.

Although the MIT rule was the starting point for derivation of the online automatic tuning method, concepts applying directly the MIT rule did not give satisfactory results. The alternatives presented in the last two sections were capable to upgrading performance.

Comparison method 2 and 3 for different $\beta$ and $\gamma$

Adaptation using the integral error as well as adaptation using squared error give similar results for $\gamma_{1}$ and $\gamma_{2}$ (Figs. $4,5)$. Both adaptation mechanisms require $5 \mathrm{~h}$ to upgrade controller performance and to cancel underdamped behavior. Controller tuning parameters $\gamma_{1}$ and $\gamma_{2}$ converge to similar values, but adaptation using the integral error is smoother.

Figure 6 shows simulations with different initialized values of the controller parameters $\gamma$. Figure $6 \mathrm{a}, \mathrm{b}$ illustrates once again the adaptation of $\gamma_{1}$ and $\gamma_{2}$ initialized with too small values. Simulations initialized close to the endvalues of the previous run (Fig. 6a, b) showed that the adaptation mechanisms leave the tuning parameters almost unchanged (Fig. 6c, d). Similar adaptation results are found for simulations initialized with large initial parameters (Fig. 6e, f). Large tuning parameters slow down the controller actions, but only marginally decrease controller performance. As a result, the driving force (or error) for adaptation is small and so is the adaptation of controller parameters.

Figure 7 shows additional simulations with different values of the adaptation rate $\beta$. Figure $7 \mathrm{c}, \mathrm{d}$ illustrates once again the effect of the chosen adaptation rate $\beta$ on $\mu$ and $\gamma$ as a reference. Small values for the adaptation rate $\beta$ give slow adaptation of controller parameters $\gamma$ (Fig. 7a, b). The oscillations are attenuated two to three times slower compared with the chosen $\beta$ and about two times faster relative to fixed $\gamma(\beta=0$, compare with Fig. 3). If $\beta$ is chosen
Fig. 6 Tuning parameters $\gamma_{1}$ and $\gamma_{2}$ for different initializations of $\gamma$. Fed-batch start at $t=22 \mathrm{~h}$. a, c, e Method 2 (integral errors, Eq. 23, $\left.\beta_{2}=225, \beta_{3}=550\right) . \mathbf{b}, \mathbf{d}, \mathbf{f}$ Method 3 (squared errors Eq. $\left.24, \beta_{1}=0.25, \beta_{2}=0.5\right)$. a, b $\gamma_{1}$ and $\gamma_{2}$ initialized too small. c, $\mathbf{d} \gamma_{1}$ and $\gamma_{2}$ initialized close to the end-values of the previous run. e, $\mathbf{f} \gamma_{1}$ and $\gamma_{2}$ initialized too large
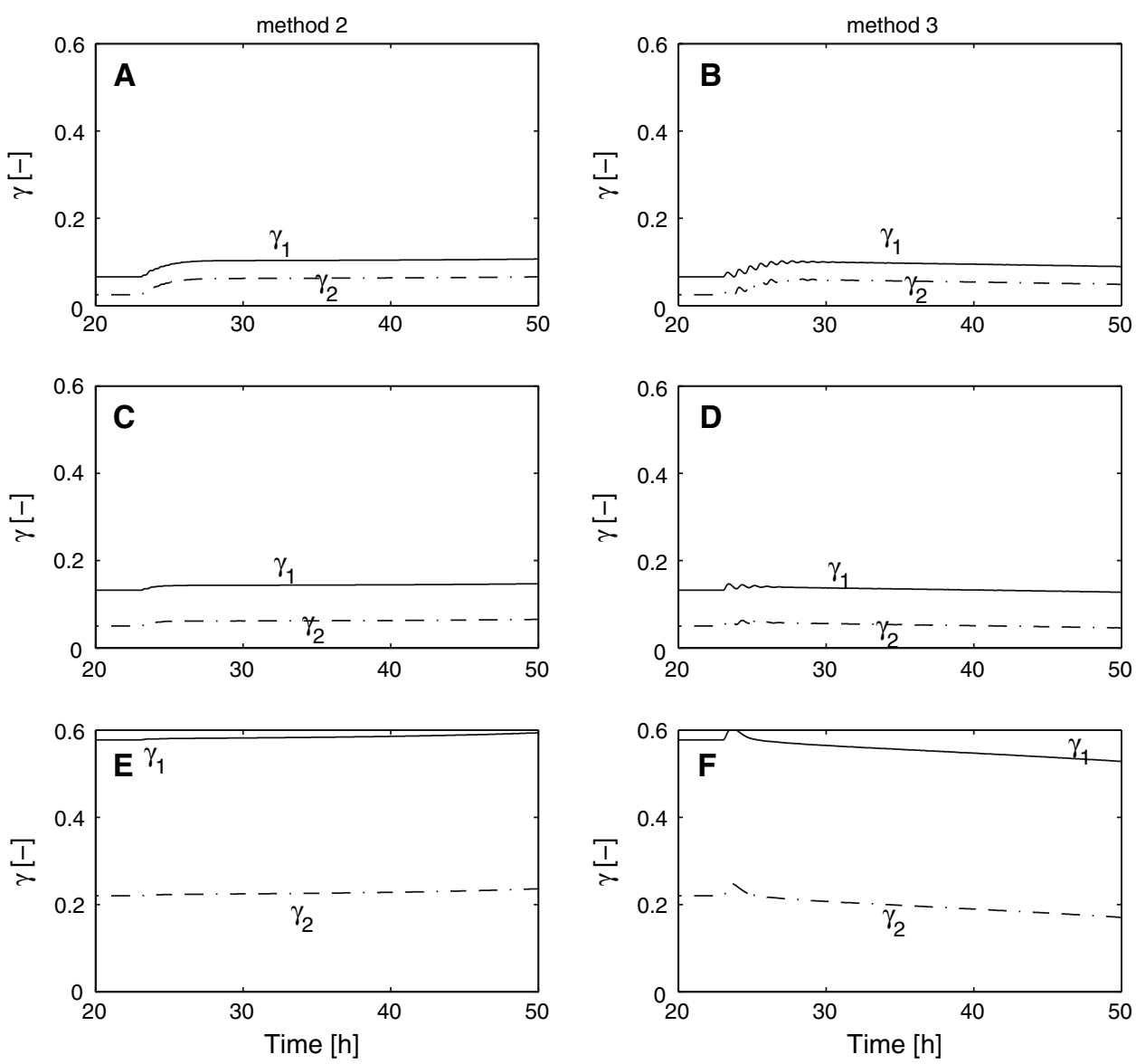
Fig. 7 The effect of different $\beta$ values on adaptation of tuning parameters $\gamma_{1}$ and $\gamma_{2}$ (\{ $\{$ it right axis $\})$ and on $\mu$ (\{lit left axis $\})$. Fed-batch start at $t=22 \mathrm{~h}$. c, d $\beta$ initialized with the chosen values. c $\beta_{2}=225, \beta_{3}=550$. d $\beta_{1}=0.25, \beta_{2}=0.5$. a, $\mathbf{c}, \mathbf{e}$ Method 2 (integral errors, Eq. 23). b, d, f Method 3 (squared errors Eq. 24). a, b $\beta$ initialized ten times smaller. $\mathbf{e}, \mathbf{f}$ $\beta$ initialized ten times larger
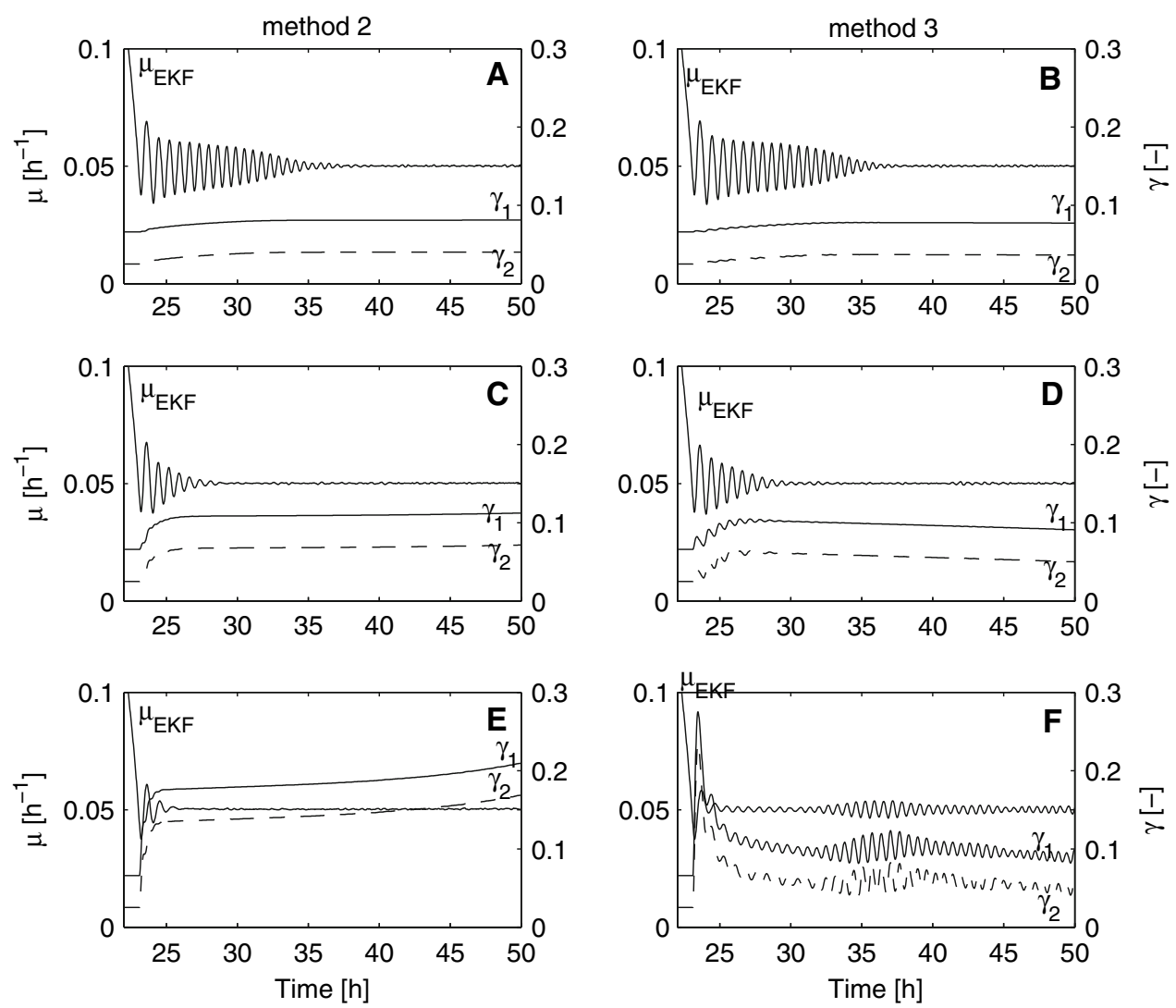

larger, adaptation becomes faster and larger values for $\gamma$ are obtained (Fig. 7e). Large tuning parameters slow down the controller actions and may give small offsets. The adaptation rate $\beta$ in Fig. $7 \mathrm{f}$ is chosen too large and gives interference with other dynamics (dissolved oxygen and specific growth rate). So, if the adaptation rate $\beta$ is not chosen too fast, both methods show robust adaptation.

\section{Stability}

In previous sections the automatic tuning mechanisms were evaluated by simulations. Although performance is good the effect of changing the tuning parameters on stability is yet unknown. Bastin and Dochain [31] state that stability of fed-batch cultivations is not an issue because the exponentially increasing biomass and volume make the system inherent unstable and result in positive poles. However, because the positive poles can be cancelled in the controller loops it makes sense to examine stability properties of the control loops.

Dagci et al. [6] "solve" the stability issue by examining the stability of the control loop; others $[4,5,16]$ refer to the stability of the reference model. In this work, stability is examined by considering the poles of the closed loop. The full system is given by Eqs. 3, 4, 7-9, 12, and the derivative of Eq. 1 including the controller Eqs. 13 and 15. The following time-varying state space model represents the linearized form of this system along the trajectory of fedbatch cultivation (see Appendix 2 for the equations and the linearization of the system):

$$
\begin{aligned}
& \frac{\mathrm{d} x}{\mathrm{~d} t}=A(t) x+B(t) u \\
& y=C(t) x \\
& u=F(t) x
\end{aligned}
$$

with $x$ the state variables, $y$ the output variables, $u$ the input variables, $A$ the system matrix, $B$ the input matrix, $C$ the output matrix, and $F$ the state feedback matrix.

The closed loop is stable if the poles of the matrix $(A+B F)$ are in the left half plane. The poles are calculated numerically for each time instant and each set of tuning parameters $\gamma_{1}$ and $\gamma_{2}$ during the simulation experiments. Figure 8 gives an illustration of the effect of $\gamma_{1}$ and $\gamma_{2}$ on closed loop stability and on controller performance for a range (250 data points between $1.65 \times 10^{-5}$ to 1.03 for $\gamma_{1}$ and $6.5 \times 10^{-6}$ to 0.41 for $\left.\gamma_{2}\right)$, where the ratio $\gamma_{1} / \gamma_{2}$ is fixed at one point of the trajectory $(t=26.7 \mathrm{~h})$. Similar results are obtained at other points along the trajectory. All real parts of the poles in Fig. 8a-e are negative. Figure $8 \mathrm{f}$ and $\mathrm{g}$ 
Fig. 8 Example of real and imaginary poles of the closed loop system for a range of $\gamma_{1}$ and $\gamma_{2}$ calculated by offline simulation of the process at one time point along the trajectory. The arrows indicate the direction in which the poles move with increasing controller tuning parameters $\gamma_{1}$ and $\gamma_{2}$
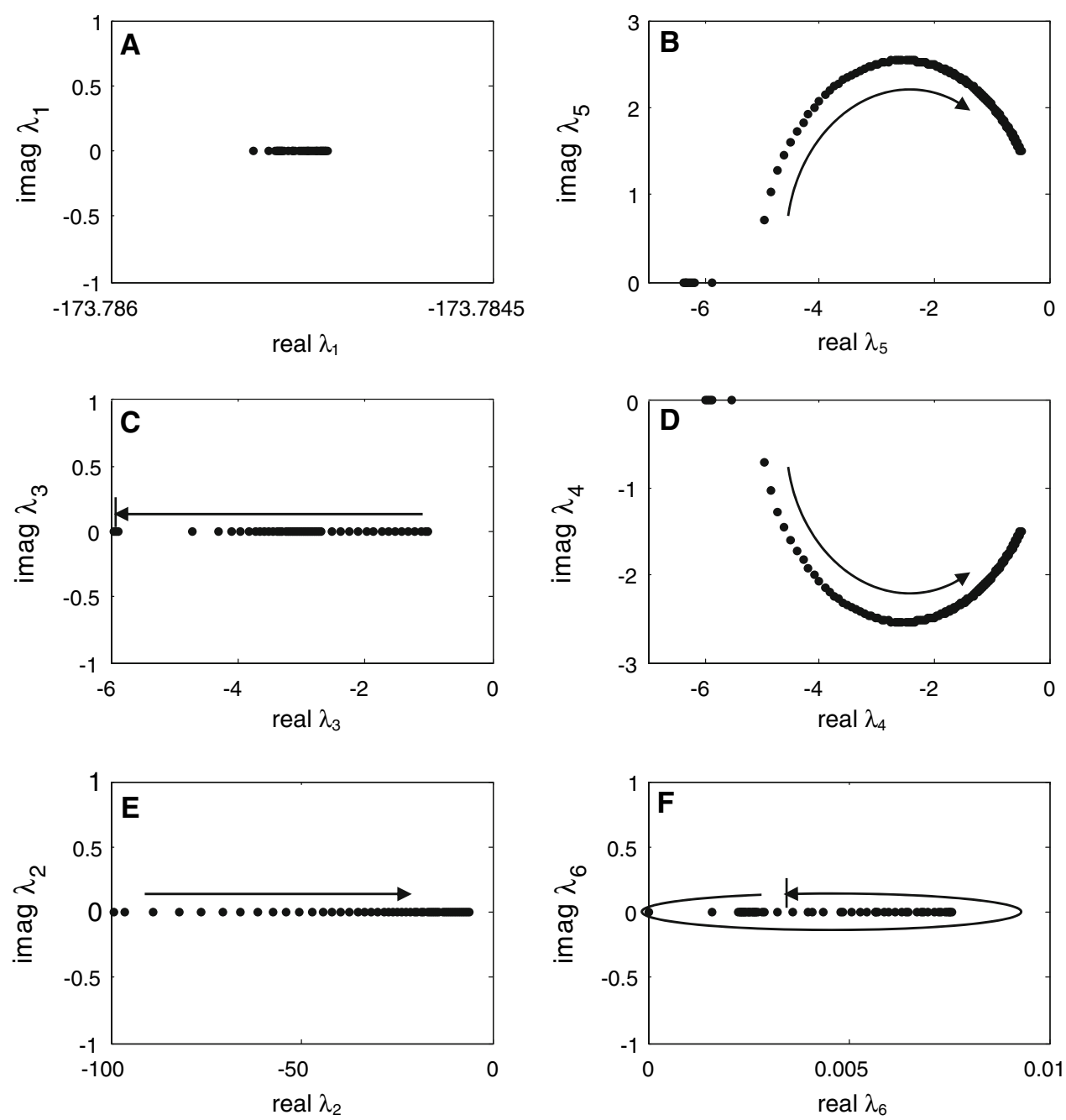

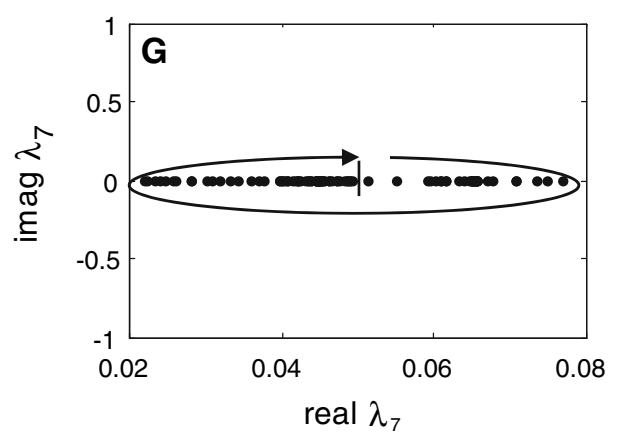

show positive poles that are dominated by the exponentially increasing biomass and volume according to the associated eigenvectors.

Small values of $\gamma$ increase the stability margin for most poles. Large values, on the other hand, result in a smooth control of the specific growth rate. Evaluation of the transfer functions $\mu(s) / \mu_{\text {set }}(s)$ and $\mathrm{DO}(s) / \mathrm{DO}_{\text {set }}(s)$ showed that the positive poles are cancelled in these transfer functions and only negative poles are relevant for these functions.

\section{Experimental results}

Controlled fed-batch cultivations with the dual substrate consuming bacterium B. pertussis (causative agent of whooping cough disease) were performed in a 7-1 laboratory bioreactor with a chemically defined medium containing glutamate and L-lactate as the main carbon sources [7]. Bioreactor conditions, analysis, and software were applied as reported in [4]. The fed-batch, the $\mu$ controller, and the automatic tuning started automatically 
when the limiting substrates were almost depleted and $\mu$ dropped to the set-point.

Control performance of the automatic tuning method and the specific growth rate controller were evaluated for the best methods of the simulations: method 2: based on the (integral) error (Eq. 23) and method 3: based on the (squared) error (Eq. 24).

Method 2: Adaptation based on the (integral) error

The automatic tuning method based on the (integral) error (Eq. 23) was implemented and tested by initializing control parameters $\gamma_{1}$ and $\gamma_{2}$ with values, which turned out to be too small in practice. In simulations it turned out that the adaptation parameter $\gamma$ is slightly increasing as the experiment proceeds toward the end. This makes the $\gamma$ correction mechanism more sensitive to errors than necessary. Therefore, in order to safeguard the experiment, it was decided to use in the experiment Eq. 26 instead of Eq. 23, which leads to down-tuning of the adaptation toward the end of the cultivation.

$\frac{\mathrm{d} \gamma_{1}}{\mathrm{~d} t}=\varepsilon \cdot\left(\beta_{2} \frac{\varepsilon}{\gamma_{1}}+\beta_{3} \int_{0}^{t} \frac{\varepsilon}{\gamma_{2}} \mathrm{~d} t\right)$

$\frac{\mathrm{d} \gamma_{2}}{\mathrm{~d} t}=\varepsilon \cdot\left(\beta_{2} \frac{\varepsilon}{\gamma_{1}}+\beta_{3} \int_{0}^{t} \frac{\varepsilon}{\gamma_{2}} \mathrm{~d} t\right)$

Evaluation after the experiment showed that the difference with the original $\gamma$ adaptation is small, so that, on retrospect, the precaution was unnecessary.

The laboratory experiment showed that by initiating the automatic tuning method at the start of the fed-batch at about 20 hours control performance was upgraded (Fig. 9). The mean absolute error and oscillation measure decrease with time. The adaptation of $\gamma_{1}$ and $\gamma_{2}$ continues during the whole fed-batch cultivation, but the changes in the first period are the strongest and at the end the values are close to the steady state.

A standard deviation of $0.005 \mathrm{~h}^{-1}$ on $\mu$ is obtained during the fed-batch phase and indicates that the long-term performance is good. The controller maintained $\mu$ close to $\mu_{\text {set }}$ in the presence of various uncertainties including disturbances on dissolved oxygen consumption, uncertain parameters, and initialization errors.

An exponentially increasing feed rate (Fig. 9d) was added into the bioreactor to cope with the exponentially increasing biomass (Fig. 9c). At $t=33.5 \mathrm{~h}$ agitation speed was increased with $100 \mathrm{rpm}$ to meet the increasing the oxygen demand during the fed-batch phase. The resulting peak in the dissolved oxygen concentration caused a disturbance in the estimated specific growth rate at $t=33.5 \mathrm{~h}$ and in the mean absolute error and oscillation error. The controller parameters were hardly adapted and the specific growth rate was properly controlled until the end of the cultivation.

At $t=40 \mathrm{~h}$ the mean absolute error and the oscillation measure became approximately constant. As an option a performance monitor can be added to the system, which can be used to evaluate the performance criteria and as a manager to (de)activate the automatic tuning method. The first function of the performance monitor is to qualify controller performance using the criteria given in Eqs. 18 and 19. Next, based on the qualification, the performance monitor decides to retune the process using an automatic tuning method or to continue with the current settings. If control performance is upgraded and the performance measures are constant, tuning is finished and the specific growth rate controller can continue with the obtained settings. In the experiment at $t=40 \mathrm{~h}$ in Fig. 9, the performance monitor would decide to continue the cultivation with the current settings for $\gamma_{1}$ and $\gamma_{2}$ and to deactivate the automatic tuning method. Subsequently, the following cultivations can be performed with the obtained settings.

\section{Method 3: Adaptation based on the (squared) error}

Figure 10 shows a part of a long-term experiment direct after initialization of the automatic tuning controller. The oscillations that occur due to an incorrect choice of $\gamma_{1}$ and $\gamma_{2}$ vanish within three hours. The offset at the end of this period disappears in some hours. The tuning method is doing its task well.

The adaptation of $\gamma_{1}$ and $\gamma_{2}$ continues and has not come to steady state after $3 \mathrm{~h}$. The oscillations that were observed in the simulations for this tuning method in the courses of $\gamma_{1}$ and $\gamma_{2}$ are hardly present in the experiment (compare Fig. 10 with Fig. 6)

Comparing the simulation (Fig. 6) with the experimental results (Fig. 10), it can be observed that the tuning parameters $\gamma_{1}$ and $\gamma_{2}$ giving the best performance during laboratory experiments are a factor five to six larger than the tuning parameters giving the best performance during simulations. Mismatches between experimental-simulation are common for biological processes. The experiments show that the automatic tuning method compensates for these mismatches by adapting the controller settings such that the desired behavior is acquired.

Calculation of the poles of the closed loop for the laboratory experiments shows that the tuning parameters are adapted into the proper direction within the stability area. 
Fig. 9 Results for laboratory experiments with automatic tuning based on (integral) errors (method 2, Eq. 26). Fed-batch start at $t=19.7 \mathrm{~h}, \beta_{2}=20$, $\beta_{3}=20$. a Oxygen uptake rate (OUR). b Specific growth rate $(\mu)$. c Biomass. d Substrate feed rate. e Controller tuning parameters. f Mean absolute error. $\mathbf{g}$ Oscillation measure
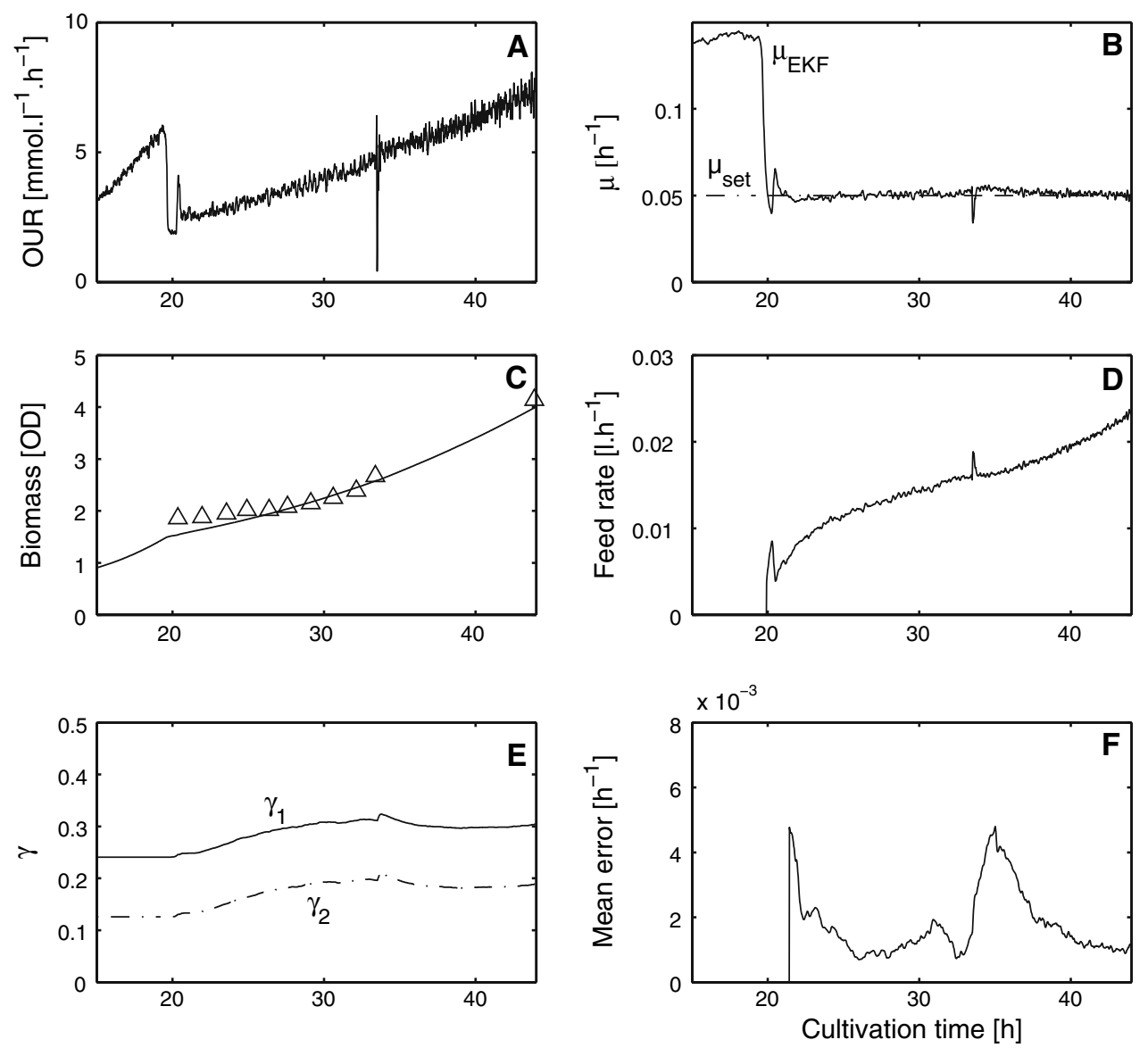

These results confirm the closed loop stability, which was already calculated for the simulated process.

\section{Conclusions}

The challenge was to upgrade performance of poorly acting controllers. Online automatic tuning has this capability. Three automatic tuning methods were applied to upgrade control performance for specific growth rate control during fed-batch cultivation. The best two methods were qualified by laboratory experiments for $B$. pertussis. In the first method, the adaptation rate is proportional to the product of the error and the integral error. The second uses an adaptation rate proportional to the error and the squared error. The methods do not require online identification, thus avoiding the need for process perturbation and complex implementation. The qualifications of the controller and automatic tuning method are:

- Control performance is evaluated online on the basis of the current mean absolute error and oscillation measure. A performance monitor can be used to activate or deactivate the automatic tuning device.

- If control performance is poor, application of automatic tuning yields good performance within $5 \mathrm{~h}$ by adapting the controller parameters such that the mean absolute error and oscillation measure decrease at least tenfold. 

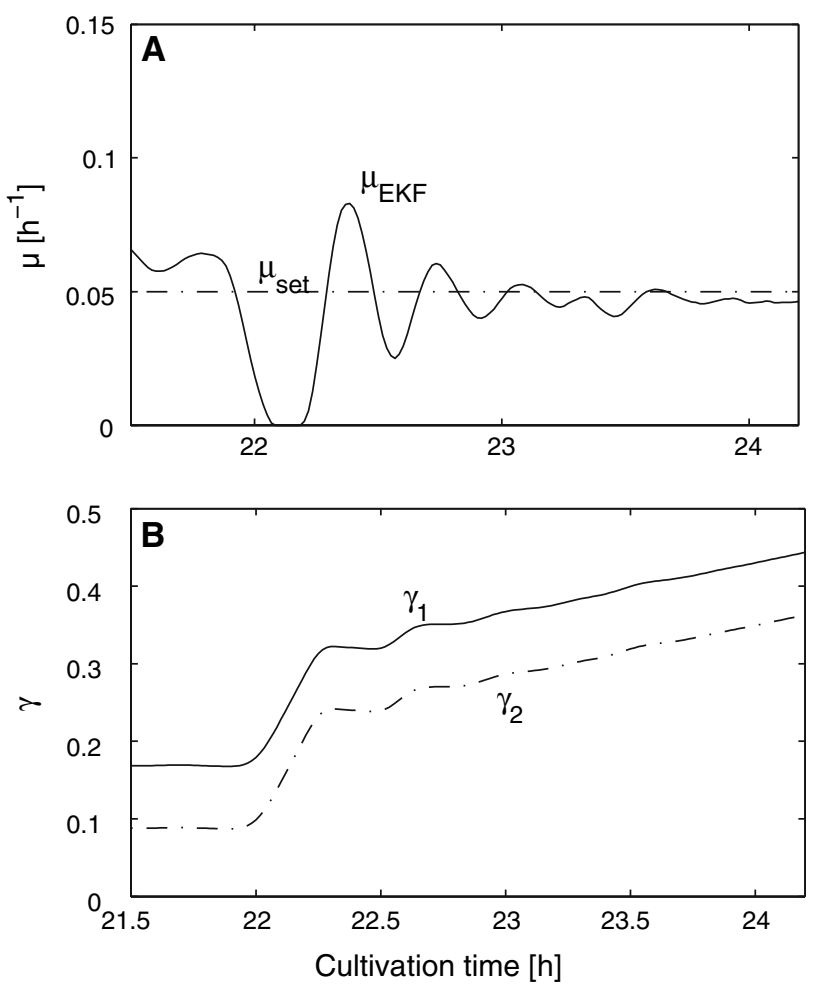

Fig. 10 Detail of results for laboratory experiments with adaptation based on the (squared) error (method 3, Eq. 24, $\beta_{1}=0.25, \beta_{2}=0.5$ ). Fed-batch start at $t=21.7 \mathrm{~h}$. a Specific growth rate $(\mu)$. b Controller tuning parameters

The automatic tuning methods are robust against disturbances among others noise, parameter uncertainties, and initialization errors.

- We expect that a cultivation controlled at the desired specific growth rate will result in smaller variations in end quality (vaccine titer) and thus yield a better product (vaccine).

- The closed loop with automatic tuning is stable at any point along the trajectory of fed-batch cultivation.

- Adaptation of control parameters is straightforward, fast and accurate. This feature, furthermore, is neither specific to fed-batch cultivation nor for specific growth rate control and hence can also be applied to other systems.

The applied automatic tuning methods improve controller performance and reduce the tuning effort by automatically adjusting the tuning parameters in one or more tuning runs.

Acknowledgments Wageningen University and the Netherlands Vaccine Institute (NVI) work together in a project with Applikon Biotechnology BV and Siemens NV in order to improve the production process by release of biopharmaceuticals on the basis of new online techniques. The Dutch Ministry of Economical Affairs (TSGE3067) funds the project. Experiments are performed at NVI.
Open Access This article is distributed under the terms of the Creative Commons Attribution Noncommercial License which permits any noncommercial use, distribution, and reproduction in any medium, provided the original author(s) and source are credited.

\section{Appendix 1: Software sensor}

Figure 11 shows that the estimated biomass coincides well with the offline biomass measurements during continuousflow stirred-tank (CSTR) cultivations. The estimated specific growth rate converged exactly to the preset dilution rate. Uncertainty on $\mu$ was small throughout the cultivation.

\section{Appendix 2: Poles and stability}

Closed loop stability is evaluated using the process equations (27)-(33).

$\frac{\mathrm{d} V}{\mathrm{~d} t}=F_{G+L}^{\mathrm{in}}$
$\frac{\mathrm{d} C_{X}}{\mathrm{~d} t}=\left(\mu-\frac{F_{G+L}^{\mathrm{in}}}{V}\right) C_{X}$
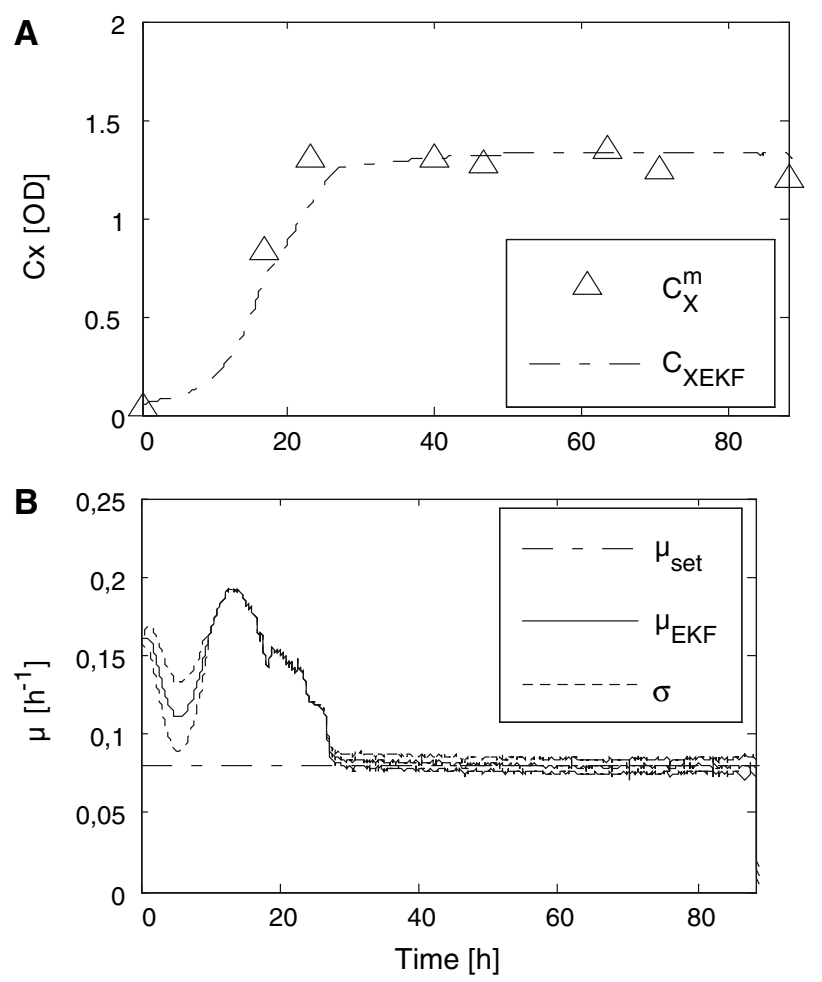

Fig. 11 Observer for CSTR cultivation. a Biomass. b Estimated specific growth rate and its standard deviation $(\sigma)$ 
$\frac{\mathrm{dDO}}{\mathrm{d} t}=k_{l} a \cdot\left(O_{2}^{\mathrm{in}}-\mathrm{DO}\right)-\left(\frac{\mu}{Y_{\mathrm{O}}}+m_{\mathrm{O}}\right) C_{X}-\frac{F_{G+L}^{\mathrm{in}}}{V} \cdot \mathrm{DO}$

$\frac{\mathrm{dDO}_{\text {sensor }}}{\mathrm{d} t}=\frac{1}{\tau_{\text {sensor }}} \cdot\left(\mathrm{DO}-\mathrm{DO}_{\text {sensor }}\right)$

Instead of the dual substrate model, the rate of change of the specific growth rate is used as forced by the input $F_{G+L}^{\text {in }}[4]$ :
$x=\left[\begin{array}{lllllll}V & C_{X} & \mathrm{DO} & \mathrm{DO}_{\text {sensor }} & \mu & x_{6} & x_{7}\end{array}\right]^{T}$

inputs:

$u=\left[\begin{array}{ll}F_{G+L}^{\text {in }} & O_{2}^{\text {in }}\end{array}\right]^{T}$

and outputs:

$y=\left[\begin{array}{ll}\mathrm{DO} & \mu\end{array}\right]^{T}$

Linearization with respect to the states at each time instant gives the Jacobian $A$ :

$A=\left[\begin{array}{ccccccc}\frac{F_{G+L}^{\mathrm{in}}}{0} \cdot C_{X} & 0 & 0 & 0 & 0 & 0 & 0 \\ \frac{F_{G+L}^{\mathrm{in}} \cdot \mathrm{DO}}{V^{2}} & \mu-\frac{F_{G+L}^{\mathrm{in}}}{V} & 0 & 0 & C_{X} & 0 & 0 \\ 0 & -\left(\frac{\mu}{Y_{\mathrm{O}}}+m_{\mathrm{O}}\right. & -\left(k_{\mathrm{L}} a+\frac{F_{G+L}^{\mathrm{in}}}{V}\right) & 0 & -\frac{C_{X}}{Y_{\mathrm{O}}} & 0 & 0 \\ -\frac{F_{G+L}^{\text {in }} \cdot\left(a C_{\mathrm{G}}^{\mathrm{in}}+b C_{\mathrm{L}}^{\mathrm{in}}\right)}{V^{2}} & 0 & \frac{1}{\tau_{\text {sensor }}} & -\frac{1}{\tau_{\text {sensor }}} & 0 & 0 & 0 \\ 0 & -(a c+b d) & 0 & 0 & 0 & 0 & 0 \\ 0 & 0 & 0 & 0 & -1 & 0 & 0 \\ 0 & 0 & 0 & -1 & 0 & 0 & 0\end{array}\right]$

$\frac{\mathrm{d} \mu}{\mathrm{d} t}=a\left(\frac{F_{G+L}^{\mathrm{in}}}{V} C_{\mathrm{G}}^{\mathrm{in}}-c C_{X}\right)+b\left(\frac{F_{G+L}^{\mathrm{in}}}{V} C_{\mathrm{L}}^{\mathrm{in}}-\mathrm{d} C_{X}\right)$

where the input of the specific growth rate controller $F_{G+L}^{\mathrm{in}}$ is given by:

$$
\begin{aligned}
F_{G+L}^{\text {in }}= & \frac{a c+b d}{a C_{\mathrm{G}}^{\text {in }}+b C_{\mathrm{L}}^{\text {in }}} \hat{C}_{X} V+\frac{V \cdot\left(\mu_{\text {set }}-\hat{\mu}\right)}{\gamma_{1}\left(a C_{\mathrm{G}}^{\text {in }}+b C_{\mathrm{L}}^{\text {in }}\right)} \\
& +\int_{0}^{t} \frac{V \cdot\left(\mu_{\text {set }}-\hat{\mu}\right)}{\gamma_{2}\left(a C_{\mathrm{G}}^{\text {in }}+b C_{\mathrm{L}}^{\text {in }}\right)} \mathrm{d} t
\end{aligned}
$$

Dissolved oxygen is controlled using standard PI control:

$$
\begin{aligned}
O_{2}^{\text {in }}= & K_{C} \cdot\left(\mathrm{DO}_{\text {set }}-\mathrm{DO}_{\text {sensor }}\right) \\
& +\int_{0}^{t} \frac{K_{C}}{\tau_{I}} \cdot\left(\mathrm{DO}_{\text {set }}-\mathrm{DO}_{\text {sensor }}\right) \mathrm{d} t+C_{\mathrm{N}}
\end{aligned}
$$

The integral parts of the controllers are added to the system by

$\frac{\mathrm{d} x_{6}}{\mathrm{~d} t}=\mu_{\mathrm{set}}-\mu$

$\frac{\mathrm{d} x_{7}}{\mathrm{~d} t}=\mathrm{DO}_{\text {set }}-\mathrm{DO}_{\text {sensor }}$

with states:
Linearization with respect to the inputs at each time instant gives $B$ :

$$
B=\left[\begin{array}{cc}
1 & 0 \\
-\frac{C_{X}}{V} & 0 \\
-\frac{\mathrm{DO}}{V} & k_{\mathrm{L}} a \\
0 & 0 \\
\frac{a C_{\mathrm{G}}^{\mathrm{in}}+b C_{\mathrm{L}}^{\mathrm{in}}}{V} & 0 \\
0 & 0 \\
0 & 0
\end{array}\right]
$$

DO and $\mu$ are the outputs:

$C=\left[\begin{array}{lllllll}0 & 0 & 0 & 1 & 0 & 0 & 0 \\ 0 & 0 & 0 & 0 & 1 & 0 & 0\end{array}\right]$

Using the controllers for specific growth rate (Eq. 32) and dissolved oxygen (33)

$$
\begin{aligned}
u & =\left[\begin{array}{c}
F_{G}^{\text {in }}+L \\
O_{2}^{\text {in }}
\end{array}\right] \\
& =\left[\begin{array}{c}
\frac{a c+b d}{a C_{\mathrm{G}}^{\text {in }}+b C_{\mathrm{L}}^{\text {in }}} C_{X} V+\frac{V \cdot\left(\mu_{\mathrm{set}}-\mu\right)}{\gamma_{1}\left(a C_{\mathrm{G}}^{\mathrm{in}}+b C_{\mathrm{L}}^{\text {in }}\right)}+\frac{V \cdot x_{6}}{\gamma_{2}\left(a C_{\mathrm{G}}^{\mathrm{in}}+b C_{\mathrm{L}}^{\text {in }}\right)} \\
C_{\mathrm{N}}+K_{C} \cdot\left(\mathrm{DO}_{\text {set }}-\mathrm{DO}_{\text {sensor }}\right)+\frac{K_{C}}{\tau_{I}} \cdot x_{7}
\end{array}\right] \cong F \cdot x
\end{aligned}
$$

the time varying state feedback is linearized with respect to the states in each operating point and becomes:

$F=\left[\begin{array}{ccccccc}\frac{a c+b d}{a C_{\mathrm{G}}^{\mathrm{i}}+b C_{\mathrm{L}}^{\mathrm{in}}} C_{X}+\frac{\left(\mu_{\mathrm{sec}}-\mu\right)}{\gamma_{1}\left(a C_{\mathrm{G}}^{\mathrm{i}}+b C_{\mathrm{L}}^{\mathrm{in}}\right)}+\frac{\gamma_{6}}{\gamma_{2}\left(a C_{\mathrm{G}}^{\mathrm{in}}+b C_{\mathrm{L}}^{\mathrm{in}}\right)} & \frac{a c+b d}{a C_{\mathrm{G}}^{\mathrm{i}}+b C_{\mathrm{L}}^{\mathrm{in}}} V & 0 & 0 & \frac{-V}{\gamma_{1}\left(a C_{\mathrm{G}}^{\mathrm{in}}+b C_{\mathrm{L}}^{\mathrm{in}}\right)} & \frac{V}{\gamma_{2}\left(a C_{\mathrm{G}}^{\mathrm{in}}+b C_{\mathrm{L}}^{\mathrm{in}}\right)} & 0 \\ 0 & 0 & 0 & -K_{C} & 0 & 0 & \frac{K_{C}}{\tau_{I}}\end{array}\right]$ 
The closed loop is stable if the poles of the matrix $(A+B F)$ are in the left half pane.

\section{References}

1. Desbourough L, Miller R (2002) Increasing customer value of industrial control performance monitoring--Honeywell's experience. In: Sixth international conference on chemical process control, AIChE Symposium Series Number 326, 98

2. Rodriguez ME, Hozbor DF, Samo AL, Ertola R, Yantorno OM (1994) Effect of dilution rate on the release of pertussis toxin and lipopolysaccharide of Bordetella pertussis. J Ind Microbiol 13:273-278 doi:10.1007/BF01569728

3. Licari P, Siber GR, Swartz R (1991) Production of cell mass and Pertussis toxin by Bordetella pertussis. J Biotechnol 20:117-130 doi:10.1016/0168-1656(91)90221-G

4. Soons ZITA, Voogt JA, Van Straten G, Van Boxtel AJB (2006) Constant specific growth rate in fed-batch cultivation of Bordetella pertussis using adaptive control. J Biotechnol 125:252-268 doi:10.1016/j.jbiotec.2006.03.005

5. Soons ZITA, Van Straten G, Van Boxtel AJB (2006) Automatic tuning and adaptation for specific growth rate control of fed-batch cultivation. In: Conference proceedings CPC7, Lake Louise, Canada

6. Dagci OH, Efe MO, Kaynak O, Yu X (2001) Variable structure systems of biochemical processes. In: Proceedings of ISIE 2001, Pusan, Korea, pp 1690-1695

7. Thalen M, Van Den IJssel J, Jiskoot W, Zomer B, Roholl P, De Gooijer C, Beuvery C, Tramper H (1999) Rational medium design for Bordetella pertussis; basic metabolism. J Biotechnol 75:147-159 doi:10.1016/S0168-1656(99)00155-8

8. Neeleman R, Joerink M, Van Boxtel AJB (2001) Dual substrate utilisation by Bordetella pertussis. Appl Microbiol Biotechnol 57:489-493 doi:10.1007/s002530100811

9. Neeleman R (2002) Biomass performance-monitoring and control in bio-pharmaceutical production. Wageningen University, The Netherlands $\mathrm{PhD}$ thesis

10. Neeleman R, Beuvery C, Vries D, Van Straten G, Van Boxtel AJB (2004) Dual-substrate feedback control of the specific growth-rate in vaccine production. CAB9, Nancy

11. Wang NS, Stephanopoulos GN (1984) Computer applications to fermentation processes. CRC Crit Rev Biotechnol 2:1-90

12. Soons ZITA, Shi J, Van der Pol LA, Van Straten G, Van Boxtel AJB (2007) Biomass growth and $k_{L} a$ estimation using online and offline measurements. CAB10(Computer Applications in Biotechnology), Cancun

13. Babuška R, Damen MR, Hellinga C, Maarleveld H (2003) Intelligent adaptive control of bioreactors. J Intell Manuf 14: 255-265 doi:10.1023/A:1022963716905

14. Åström KJ, Wittenmark B (1995) Adaptive control. AddisonWesley, USA
15. Van Impe JF, Bastin G (1995) Optimal adaptive control of fedbatch fermentation processes. Control Eng Practice 3:939-954, doi:10.1016/0967-0661(95)00077-8

16. Smets IY, Bastin GP, Van Impe JF (2002) Feedback stabilization of fed-batch bioreactors: non-monotonic growth kinetics. Biotechnol Prog 18:116-125, doi:10.1021/bp010191p

17. Smets IY, Claes JE, November EJ, Bastin GP, Van Impe JF (2004) Optimal adaptive control of (bio)chemical reactors; past, present and future. J Process contr 14:795-805, doi:10.1016/ j.jprocont.2003.12.005

18. Chang D (2003) The snowball effect in fed-batch bioreactors. Biotechnol Prog 19:1064-1070, doi:10.1021/bp025792a

19. Levisauskas D, Simutis R, Borvitz D, Lübbert A (1996) Automatic control of the specific growth rate in fed-batch cultivation processes based on exhaust gas analysis. Bioprocess Eng 15:145150, doi:10.1007/BF00369618

20. Ignatova M, Lubenova V, Georgieva P (2000) MIMO adaptive linearizing control of fed-batch amino acids simultaneous production. Bioprocess Eng 22:79-84, doi:10.1007/PL00009106

21. Picó-Marco E, Picó J, De Battista H, Navarro JL (2004) Robust adaptive control in biotechnological fed-batch processes. CAB9, Nancy

22. Picó-Marco E, Picó J, De Battista H (2005). Sliding mode scheme for adaptive specific growth rate control in biotechnological fed-batch processes. Int $\mathbf{J}$ Control 78:128-141, doi: 10.1080/ 002071705000073772

23. Zlateva $\mathrm{P}$ (1997) Sliding-mode control of fermentation processes. Bioprocess Eng 16:383-387, doi: 10.1007/s004490050340

24. Krastanov MI, Dimitrova NS (2003) Stabilizing feedback of a nonlinear process involving uncertain data. Bioprocess Biosyst Eng 25: 217-220, doi: 10.1007/s00449-002-0299-4

25. Akay B, Ertunç S, Kahvecioğlu A, Hapoğlu H, Alpbaz M (2002) Adaptive control of $S$. cerevisae production. Trans IchemE 80:28-38, doi: 10.1205/096030802753479089

26. Åström KJ, Hägglund T (1988) Automatic PID tuning. Instrument Society of America, USA

27. Frahm B, Lane P, Atzert H, Munack A, Hoffmann M, Hass VC, Pörtner R (2002) Adaptive, model-based control by the openloop-feedback-optimal (OLFO) controller for the effective fedbatch cultivation of hybridoma cells. Biotechnol Prog 18:10951103, doi: $10.1021 / \mathrm{bp} 020035 \mathrm{y}$

28. Åström KJ, Hägglund T, Hang CC, Ho WK (1993) Automatic tuning and adaptation for PID controllers-a survey. Control Eng Prac 1:699-714, doi: 10.1016/0967-0661(93)91394-C

29. Chen L, Bastin G, Van Breusegem V (1995) A case study of adaptive nonlinear regulation of fed-batch biological reactors. Automatica 31: 55-65, doi: 10.1016/0005-1098(94)00068-T

30. Rocha I, Ferreira EC (2002) Model-based adaptive control of acetate concentration during the production of recombinant proteins with E. coli. In: 15th Triennial world congress, Barcelona, Spain

31. Bastin G, Dochain D (1990) On-line estimation and adaptive control of bioreactors. Elsevier, The Netherlands 